\title{
Tuning Range-Separated DFT Functionals for Accurate Orbital Energy Modeling of Conjugated Molecules
}

Ram S. Bhatta ${ }^{*}$ Giuseppe Pellicane ${ }^{\#}$ and Mesfin Tsige*

"Department of Polymer Science, The University of Akron, Ohio 44325, United States

${ }^{\#}$ School of Chemistry and Physics, University of Kwazulu-Natal, South Africa; and

National Institute for Theoretical Physics, Pietermaritzburg, South Africa.

"Corresponding authors: $\underline{\text { rsb20@uakron.edu; mtsige@uakron.edu }}$ 


\begin{abstract}
Density functional theory (DFT) calculations with range-separated (RS) functionals are important for orbital energy modeling of conjugated molecules that involve charge transfer excitation. However, the accuracy of the computed results depends on the range-separation parameter $(w)$, and the optimal values of $\mathrm{w}$ for a wide range of conjugated systems has hitherto been missing. Herein, orbital energy modeling of twelve representative conjugated molecules, that are promising electron-donors in bulk heterojunction-based organic photovoltaic devices, are benchmarked using DFT and time-dependent DFT (TD-DFT) with three RS functionals (LC-BLYP, wB97XD and CAM-B3LYP). The results using the RS functionals under consideration with default values of $w$ deviate largely from the experimental values (mean signed error (MSE) on HOMO are $2.1 \mathrm{eV}, 0.93 \mathrm{eV}$ and $1.5 \mathrm{eV}$, and MSE on vertical excitation energies are 0.47 $\mathrm{eV}, 0.55 \mathrm{eV}$ and $0.82 \mathrm{eV}$, respectively). Computation of orbital energies using tuned range-separation parameter for these RS functionals in the range of $0.05 \leq w \geq 0.5 \mathrm{Bohr}^{-1}$ indicates that $w$ plays a significant role on the accuracy of the ground and the excited state energies. We found that the accurate orbital energies of conjugated systems can be predicted using values of $w$ between 0.1 and $0.15 \mathrm{Bohr}^{-1}$, much smaller than the default values of $0.47,0.33$ and 0.20 Bohr $^{-1}$ used in LC-BLYP, CAM-B3LYP and wB97XD, respectively.
\end{abstract}

Keywords: Density functional theory; Range-separation parameter; Orbital energies; Band gap 


\section{Introduction}

Small conjugated molecules (SCMs) have gained immense interest in recent years because of their applications in organic photovoltaic (OPV) devices [1-3]. The promise of SCMs as electron donors in OPV devices derives from their structural simplicity, good control over synthetic reproducibility and low purification cost [3]. The current power conversion efficiency (PCE) of SCM-based OPV devices has reached 10.1\% [4], which is comparable to the PCE of the best polymer-based OPV devices $[5,6]$. However, the PCE of SCM-based OPV devices needs to be further improved for the practical usage by average citizens. Among the various parameters that affect the OPV device performance, the frontier orbital energies of SCMs are critical because the magnitude of the HOMO-LUMO energy gap directly affects the short circuit current, and the difference between the HOMO energy of the SCM and the LUMO energy of the acceptor affects the open-circuit voltage [7-9]. Furthermore, HOMO-HOMO as well as LUMO-LUMO offset between the SCM and the acceptor control the charge separation at the SCM/acceptor interface.

Density functional theory (DFT) and time-dependent DFT (TD-DFT) are the firstprinciples approaches for orbital energy modeling of conjugated systems with a moderate computational cost $[10,11]$. The hybrid DFT functional such as B3LYP is popular to yield reasonably accurate ground and excited state orbital energies of many conjugated systems [12]. However, the hybrid functional fails in correctly predicting the electronic transitions of conjugated systems involving charge transfer process [13]. To overcome this limitation, range-separated (RS) DFT functionals are introduced, which are increasingly employed for orbital energy modeling of conjugated systems [13-15]. In RS 
functionals, exchange densities are mixed non-uniformly by partitioning the Coulomb operator into the short-range and long-range components with the help of the error function, $\operatorname{erf}(w r)$, as [14]

$$
\frac{1}{r}=\frac{e r f(w r)}{r}+\frac{1-e r f(w r)}{r} .
$$

The RS functionals contain a range-separated parameter $\mathrm{w}$ in units of Bohr ${ }^{-1}$ in such a way that DFT exchange switches to zero and Hartree-Fock exchange switches to full at w. In computational packages such as Gaussian 09 [16], the default values of w for RS functioanls such as CAM-B3LYP [17], LC-BLYP [18] and wB97XD [19] are set to some specific default values (see next section). These RS functionals with default values of $w$ consistently give less negative (higher lying) LUMO energies, more negative (lower lying) HOMO energies, high HOMO-LUMO gap and high vertical excitation energies of conjugated systems compared to experimental values $[12,20]$. Recently reported studies on optimizing $\mathrm{w}$ have aimed either satisfying the Koopmans' theorem [21-23] or reproducing high-level ab initio data [24-28]. The optimal values of $w$ for range of conjugated systems are not well justified yet and the lack of these optimal values is responsible for the discrepancy between computed and experimental orbital energies. Therefore, tuning of $w$ is crucial to determine its optimal value, which is necessary for the accurate orbital energy modeling of SCMs.

In this work, we perform systematic DFT and TD-DFT calculations of orbital energies of conjugated molecules using RS functionals with tuned range-separated parameters. We compute HOMO, LUMO, HOMO-LUMO gap and vertical excitation energies. We compare the computed results with available experimental data to determine the optimum value of the range-separation parameter for each RS functional. 
We further compare the results of RS functionals with those obtained from the popular B3LYP functional.

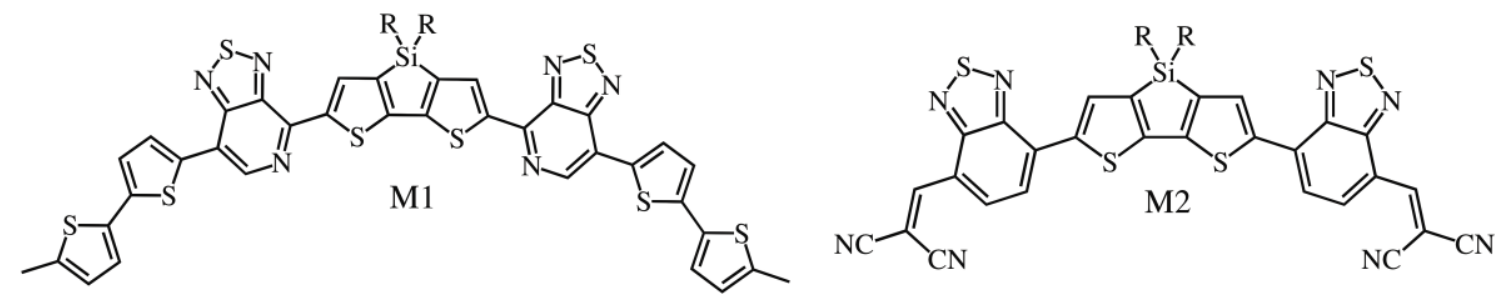<smiles></smiles>

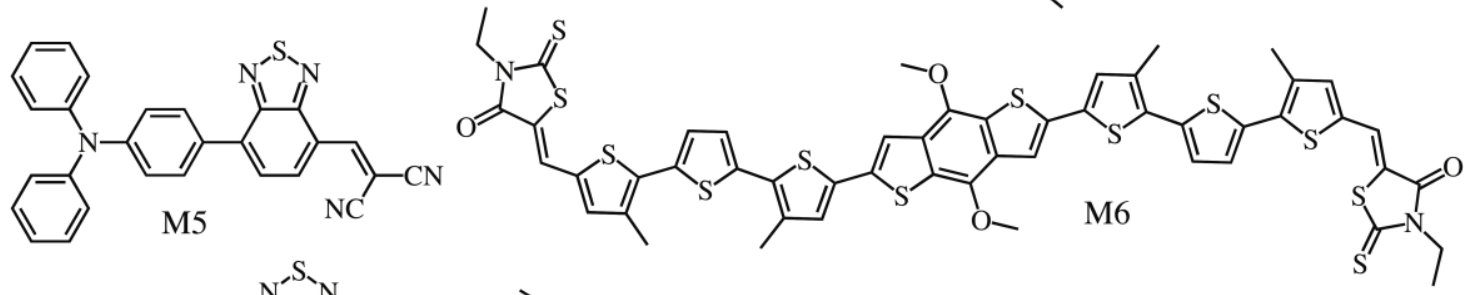<smiles>CC(C)=Cc1ccc(-c2ccc(C(c3ccc(C)cc3)(c3ccc(C)cc3)c3ccc(C)cc3)nn2)cc1</smiles>

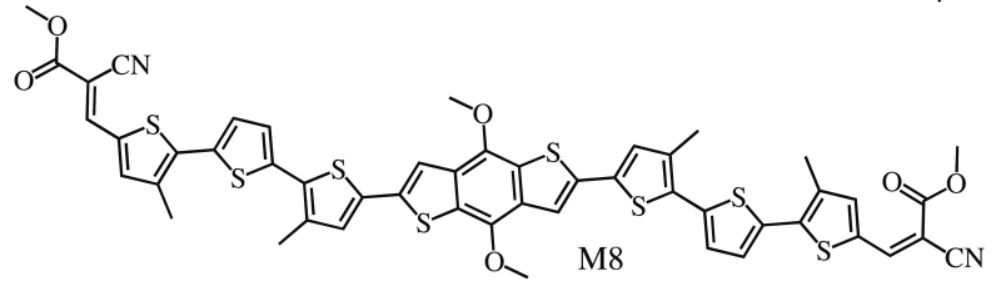<smiles>CC(N)=CC1=NC=NC1c1ccc(-c2ccc(-c3ccccc3)s2)cc1</smiles>

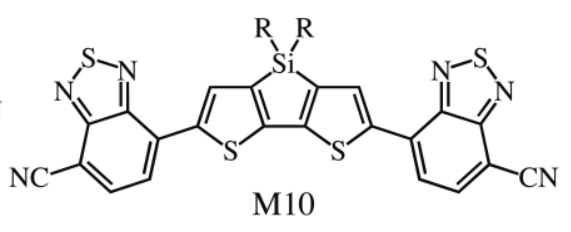<smiles>c1ccc2cc3cc4ccccc4cc3cc2c1</smiles><smiles>C1=CC(c2ccc(-c3ccc(-c4ccc(-c5cccs5)s4)s3)s2)C=C1c1cccs1</smiles>

Figure 1. Schematic representation of selected conjugated molecules in this study. Throughout the text,

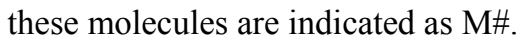




\section{Computational Methods}

In this study, representative conjugated molecules with linear and non-linear backbones, homo- and hetero donor-acceptor units, and heteroatom-substituted rings, which affect the orbital energies, were chosen and are shown in Figure 1. The geometry optimizations of each of these molecules were performed using dispersion corrected Becke's three-parameter Lee-Yang-Parr (B3LYP) exchange-correlation functional combined with $6-31 \mathrm{G}^{*}$ basis set without any constraint $[29,30]$. The B3LYP functional was chosen for geometry optimization because it has been proven from our previous studies that it produces accurate geometries of conjugated systems [31-33]. Three different RS functionals such as CAM-B3LYP [17], LC-BLYP [18] and wB97XD [19], which have default $\mathrm{w}$ values of $0.33 \mathrm{Bohr}^{-1}, 0.47 \mathrm{Bohr}^{-1}$ and $0.20 \mathrm{Bohr}^{-1}$, respectively are chosen. The total energies as well as orbital energies were calculated with these functionals starting from the optimized geometries. The HOMO-LUMO gaps were computed from the difference between HOMO and LUMO energies. The vertical excitation energies were calculated using TD-DFT at the above-mentioned levels. Calculations with the RS functionals were performed with the default as well as the tuned values of range-separation parameter in the range of $0.05 \leq w \geq 0.5 \mathrm{Bohr}^{-1}$. The upper limit of $0.5 \mathrm{Bohr}^{-1}$ for $w$ (close to the default value of $w$ for LC-BLYP) was chosen because the default value of $w$ consistently gives higher lying LUMO and lower lying HOMO energies compared to the experimental results. All computations were performed using the G09 package [16]. 


\section{Results and Discussion}

\subsection{Frontier molecular orbital energy}

Table 1. Computed HOMO energies (eV) of different molecules. Experimental values are from Refs. 1, 4, 34-39. MSE is the calculated mean signed error.

\begin{tabular}{cccccc}
\hline Molecule & Expt. & B3LYP & CAM-B3LYP & wB97XD & LC-BLYP \\
\hline M1 & -5.20 & -4.82 & -5.95 & -6.52 & -7.15 \\
M2 & -5.80 & -5.83 & -6.86 & -7.43 & -7.98 \\
M3 & -5.43 & -5.24 & -6.38 & -6.94 & -7.55 \\
M4 & -5.51 & -4.96 & -6.05 & -6.61 & -7.20 \\
M5 & -5.50 & -5.36 & -6.52 & -7.09 & -7.71 \\
M6 & -5.02 & -5.02 & -6.12 & -6.69 & -7.29 \\
M7 & -5.30 & -5.22 & -6.31 & -6.87 & -7.46 \\
M8 & -5.04 & -5.03 & -6.12 & -6.68 & -7.27 \\
M9 & -5.35 & -5.33 & -6.43 & -6.99 & -7.59 \\
M10 & -5.40 & -5.66 & -6.77 & -7.35 & -7.93 \\
M11 & -5.30 & -4.79 & -5.97 & -6.54 & -7.20 \\
M12 & -5.40 & -4.86 & -5.99 & -6.58 & -7.10 \\
MSE & & 0.18 & 0.93 & 1.5 & 2.1 \\
\hline
\end{tabular}

The computed HOMO energies for the twelve molecules using different DFT functionals and the corresponding experimental data [1,4,34-39] are presented in Table 1. The tabulated energies for RS functionals are at the default values of $\mathrm{w}$ (see previous section). It is evident from Table 1 that the computed HOMO energies using B3LYP, LCBLYP, CAM-B3LYP and wB97XD are significantly different from each other. We found that B3LYP consistently gives better correlation to experimental HOMO energies than the other range-separated functionals. This is apparent from the calculated mean signed error (MSE) values of $0.18,-2.1,-0.93$ and $-1.5 \mathrm{eV}$ for B3LYP, LC-BLYP, CAM-B3LYP and wB97XD, respectively. The MSE value for each functional was determined by average difference between the calculated energies and the experimental values. The present computed results clearly show a better performance of B3LYP as compared to CAM-B3LYP, similarly as it was found on similar conjugated systems reported by 
McCormick et al [12] $(\mathrm{MSE}=0.22 \mathrm{eV}$ and $\mathrm{MSE}=-1.07 \mathrm{eV}$ for B3LYP and CAMB3LYP, respectively).

Table 2. LUMO energies in eV. Experimental values are from Refs. 1, 4, 34-39.

\begin{tabular}{cccccc}
\hline Molecule & Expt. & B3LYP & CAM-B3LYP & wB97XD & LC-BLYP \\
\hline M1 & -3.7 & -3.08 & -1.91 & -1.42 & -1.03 \\
M2 & -3.92 & -3.87 & -2.19 & -1.69 & -1.39 \\
M3 & -3.35 & -3.22 & -1.86 & -1.36 & -0.97 \\
M4 & -3.74 & -2.87 & -1.87 & -1.37 & -0.97 \\
M5 & -3.36 & -3.28 & -2.44 & -1.94 & -1.59 \\
M6 & -3.27 & -2.83 & -2.94 & -2.45 & -2.11 \\
M7 & -3.44 & -3.15 & -1.19 & -0.67 & -0.29 \\
M8 & -3.2 & -2.84 & -1.03 & -0.50 & -0.14 \\
M9 & -3.44 & -3.22 & -2.34 & -1.84 & -1.48 \\
M10 & -3.23 & -3.42 & -2.30 & -1.80 & -1.45 \\
M11 & -3.1 & -2.18 & -2.33 & -1.84 & -1.53 \\
M12 & -2.4 & -2.07 & -2.27 & -1.78 & -1.48 \\
MSE & & 0.34 & 1.29 & 1.79 & 2.14 \\
\hline
\end{tabular}

As found for the HOMO energies, the LUMO energies are also dependent on the nature of DFT functionals. The computed as well as the corresponding experimental values of the LUMO energies are shown in Table 2. Some of the experimental values reported in Table 2 are estimated from the difference between the optical gaps and the HOMO energies and hence, they are not directly comparable to the computed LUMO energies. Nevertheless, these experimental values are helpful to provide the approximate comparison since the errors are expected to be small $(\leq 0.1 \mathrm{eV})[1,36]$. The LUMO energies computed at the B3LYP level are better correlated $(\mathrm{MSE}=0.34 \mathrm{eV})$ with the experimental LUMO energies compared to the energies computed using RS functionals. MSE values for LC-BLYP, CAM-B3LYP and wB97XD are 2.1, 1.29 and $1.79 \mathrm{eV}$, respectively. RS functionals with default w consistently give virtual orbital that are lying far higher than B3LYP and experiment. 
Since the RS functionals with the default values of $w$ give far lower lying HOMO and far higher lying LUMO energies, there is interest in finding the optimal values of $w$ in order to compute the accurate orbital energies. This can be achieved by orbital energy calculations using manually tuned values of $w$. In the present calculations, the value of $w$ for each of the RS fucntionals is systematically varied from $0.05 \mathrm{Bohr}^{-1}$ to $0.5 \mathrm{Bohr}^{-1}$ and orbital energies are calculated.

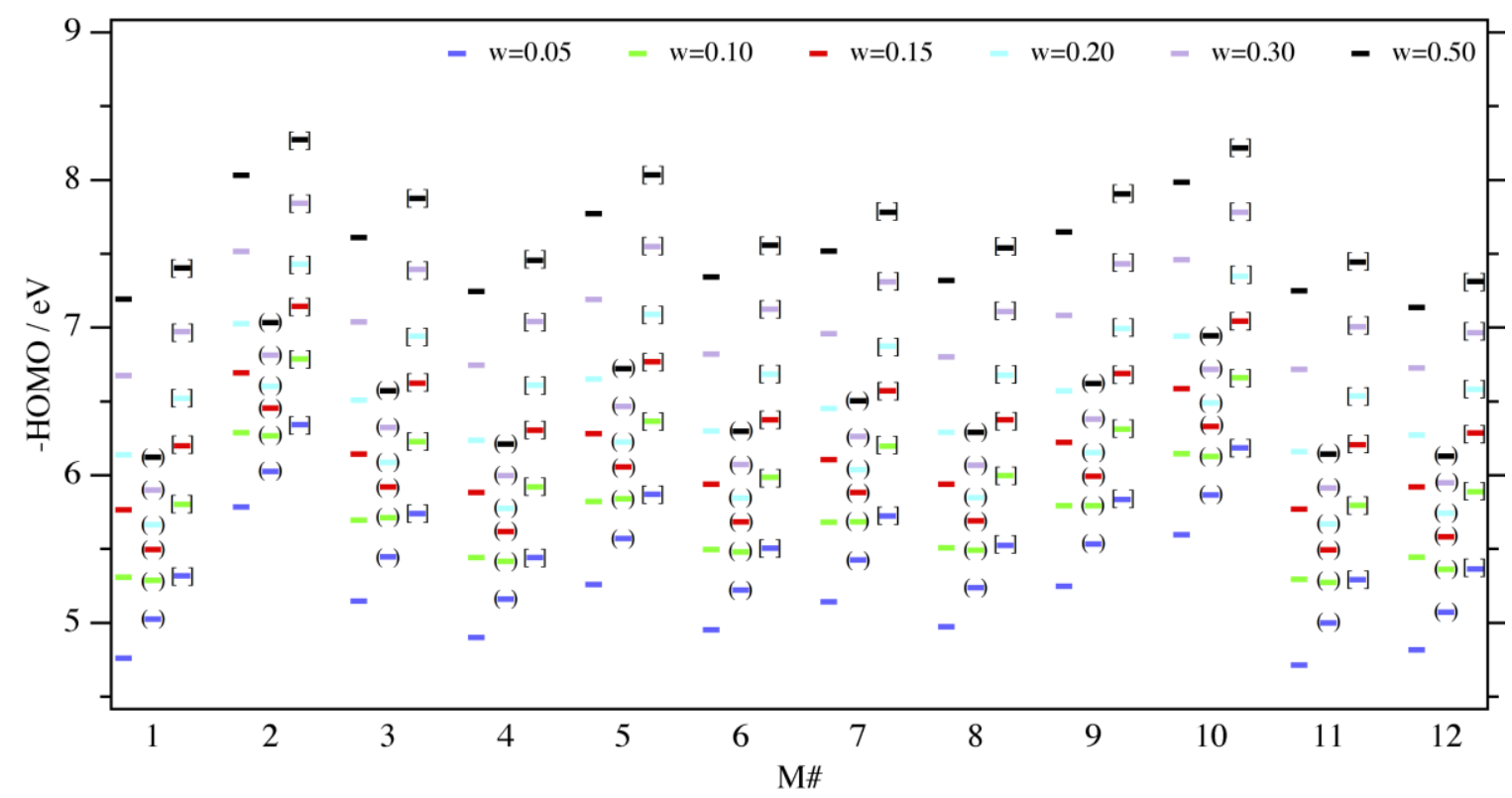

Figure 2. HOMO energies of molecules calculated at different values of $w$, where $\mathrm{M} \#$ is the molecular number shown in Figure 1. LC-BLYP, CAM-B3LYP and wB97XD energies are represented as bars, bars in parenthesis and bars in square brackets, respectively.

Figure 2 shows the HOMO energies for the twelve molecules computed using LC-BLYP, CAM-B3LYP and wB97XD RS functionals at differently tuned values of $w$. The average increase in the HOMO energies of molecules is $\sim 2.39 \mathrm{eV}$ when the value of $w$ is tuned from 0.05 to $0.5 \mathrm{Bohr}^{-1}$ for LC-BLYP, the lowest increase of $\sim 2.24 \mathrm{eV}$ being 
for M2 (planar backbone) and the highest increase of $\sim 2.53 \mathrm{eV}$ for M9 (non-planar backbone). When $\mathrm{w}<0.1 \mathrm{Bohr}^{-1}$, LC-BLYP gives slightly higher HOMO energies compared to the experimental values. LC-BLYP gives HOMO energies that are comparable to the B3LYP energies when $\mathrm{w}$ approaches $\sim 0.05 \mathrm{Bohr}^{-1}$ (MSE $\sim 0.24 \mathrm{eV}$ ). When $w>0.1$, it gives lower HOMO energies compared to the experimental values. LCBLYP gives far low-lying HOMO orbitals having energies as low as $\sim 2.58 \mathrm{eV}$ compared to the experimental energies as $\mathrm{w}$ approaches $0.5 \mathrm{Bohr}^{-1}$. The wB97XD functional follows a similar trend with the average increase in the HOMO energies by $\sim 2.05 \mathrm{eV}$. It gives low-lying HOMO energies with the increase in w and the HOMO energies reach up to $\sim 2.8 \mathrm{eV}$ below the experimental values when $w=0.5 \mathrm{Bohr}^{-1}$. The CAM-B3LYP functional shows the smallest average increase $(\sim 1.08 \mathrm{eV})$ in the HOMO energies when $w$ is tuned in the range of 0.5 to $0.05 \mathrm{Bohr}^{-1}$.

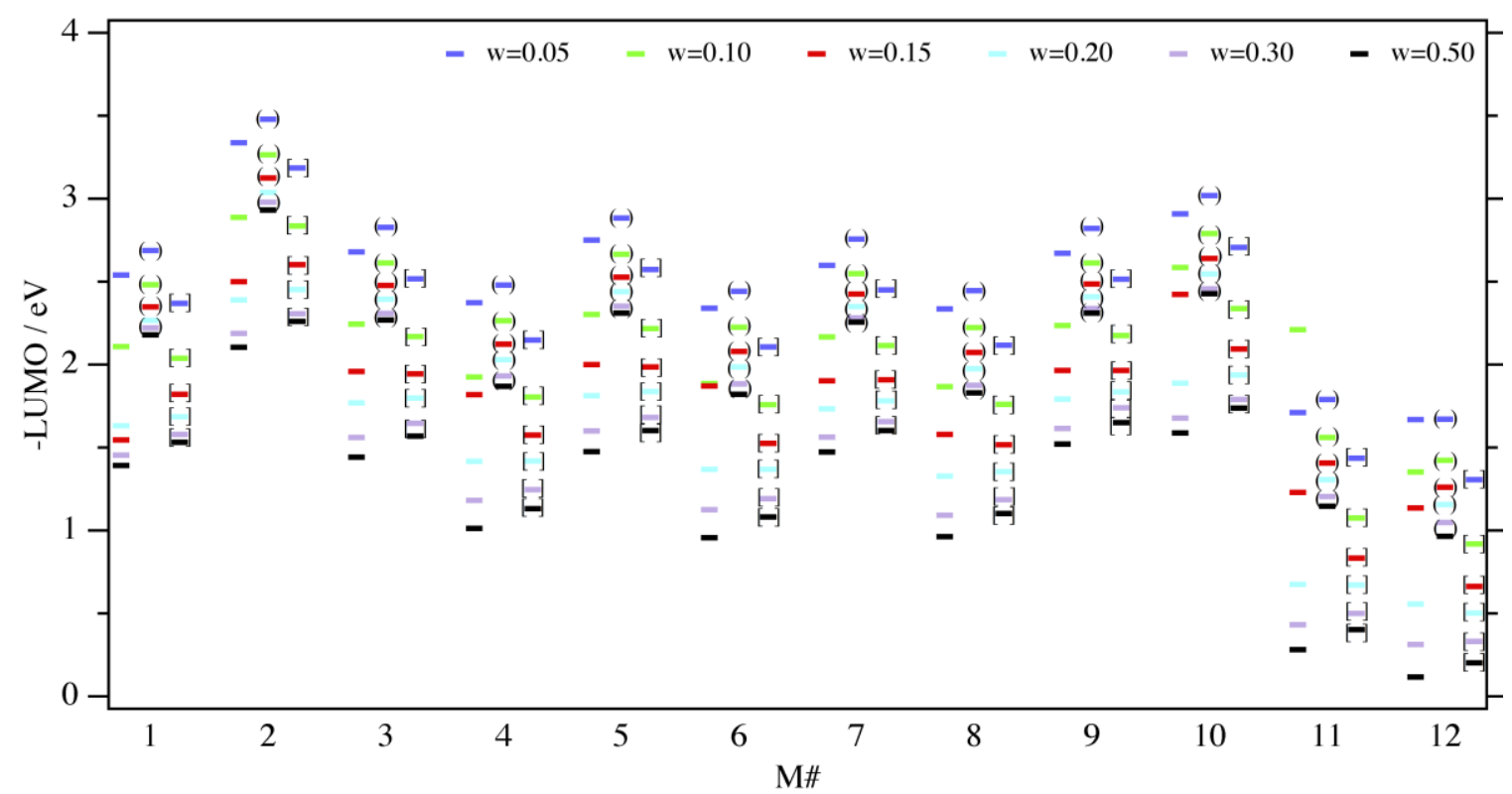

Figure 3. LUMO energies of molecules calculated at different values of $w$. LC-BLYP, CAM-B3LYP and wB97XD energies are represented as bars, bars in parenthesis and bars in square brackets, respectively. 
Tuning the value of w not only affects the HOMO energies, but also the LUMO energies. Figure 3 shows the dependence of the LUMO energies of molecules with $\mathrm{w}$ for LC-BLYP, CAM-B3LYP and wB97XD RS functionals. At the higher values of $w$ all RS functionals favor higher-lying (more positive energies) virtual molecular orbitals. These virtual molecular orbitals become low-lying with the decrease in energies as the value of $w$ decreases. The average decrease in the LUMO energies is $\sim 1.30 \mathrm{eV}$ for LC-BLYP when the value of $w$ is varied from 0.5 to $0.05 \mathrm{Bohr}^{-1}$. Both CAM-B3LYP and wB97XD follow the similar trends, but with different magnitudes. The average decrease in the LUMO energies for CAM-B3LYP and wB97XD are $0.58 \mathrm{eV}$ and $0.98 \mathrm{eV}$, respectively. The energies follow the order of LC-BLYP > wB97XD > CAM-B3LYP.

\subsection{The HOMO-LUMO Gap}

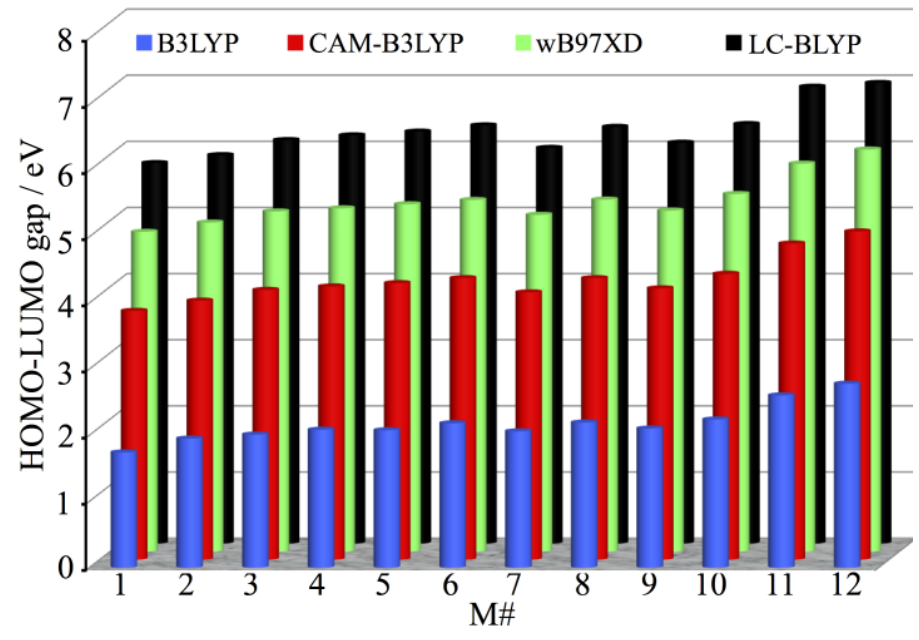

Figure 4. Computed HOMO-LUMO gaps using default RS functionals.

The RS functionals with the default values of $\mathrm{w}$ favor more negative HOMO energies and less negative LUMO energies compared to the experimental values (Tables 
1 and 2). Consequently, the HOMO-LUMO gaps of molecules are overestimated across these functionals. These gaps represent the energy difference between the valence and the conduction bands. Figure 4 summarizes the computed HOMO-LUMO gaps of molecules using B3LYP as well as RS functionals with the default values of w. The HOMO-LUMO energies follow the order of LC-BLYP $>$ wB97XD $>$ CAM-B3LYP $>$ B3LYP. In terms of the magnitude, CAM-B3LYP overestimates the HOMO-LUMO gaps by $\sim 2 \mathrm{eV}$ compared to B3LYP. Similarly, LC-BLYP and wB97XD overestimate the HOMOLUMO gaps by $\sim 3 \mathrm{eV}$ and $\sim 4 \mathrm{eV}$, respectively, compared to B3LYP. This shows that RS functionals such as CAM-B3LYP, LC-BLYP and wB97XD with the default values of $w$ overestimate the HOMO-LUMO gaps by a factor of 2 or more.

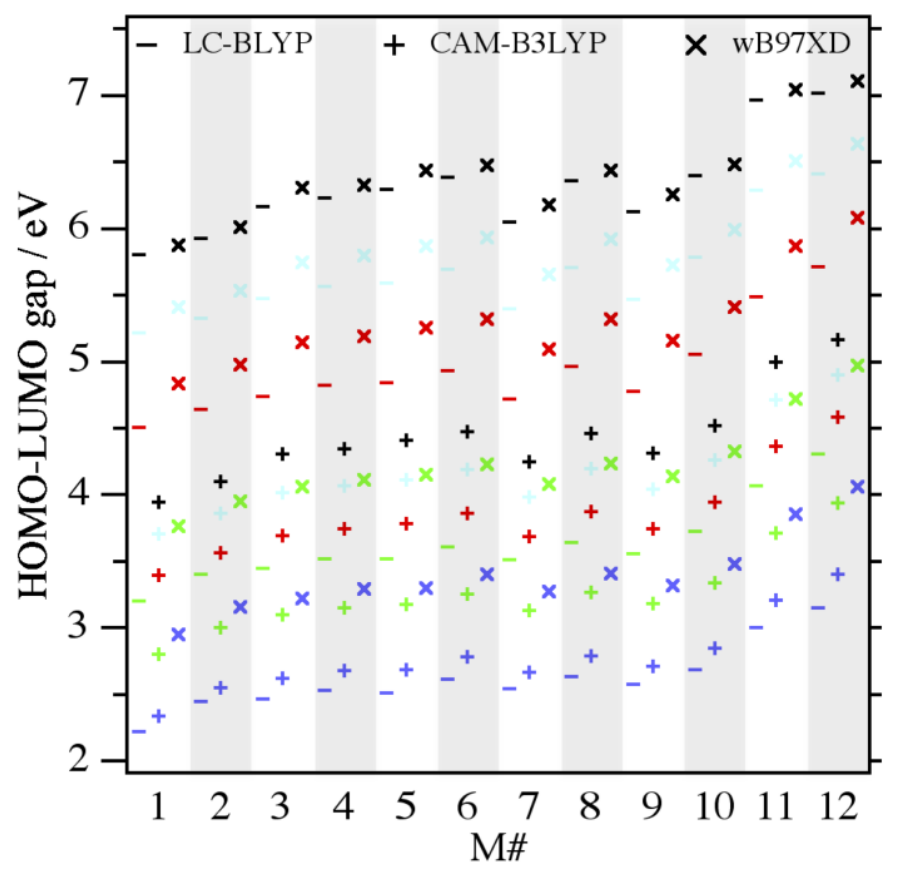

Figure 5. HOMO-LUMO gaps of molecules calculated at different values of $w$. Different colors represent different values of $\mathrm{w}$ (blue $=0.05$, green $=0.1$, red $=0.2$, cyan $=0.4$, black $=0.5$, respectively). 
The overestimation of the HOMO-LUMO gaps by the default RS functionals can be eliminated by manually tuning values of $w$. Figure 5 shows the HOMO-LUMO gaps of molecules computed using LC-BLYP, CAM-B3LYP and wB97XD RS functionals with tuned values of $w$ in the range of 0.05 to $0.5 \mathrm{Bohr}^{-1}$. We found that the average decrease in the HOMO-LUMO energies is $\sim 3.7 \mathrm{eV}$ for LC-BLYP when the value of $w$ is tuned from 0.5 to $0.05 \mathrm{Bohr}^{-1}$. The wB97XD functional favors slightly smaller decrease in the HOMO-LUMO gaps $(\sim 3 \mathrm{eV})$ compared to LC-BLYP when $w$ is tuned in a similar way. The smallest decrease in the HOMO-LUMO gaps is found for CAM-B3LYP with the average value of $\sim 1.6 \mathrm{eV}$ while decreasing the value of $w$ from 0.5 to $0.05 \mathrm{Bohr}^{-1}$. The overall trend in the decrease in the HOMO-LUMO energies follow the order of LCBLYP $>$ wB97XD $>$ CAM-B3LYP across the molecules as a result of tuning the value of $w$.

\subsection{The Optical Gaps}

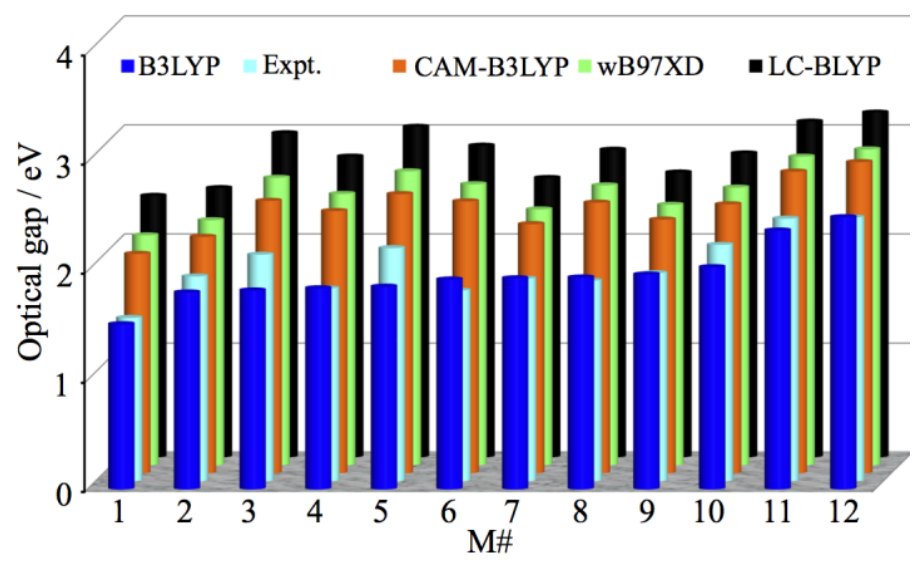

Figure 6. Computed optical gaps using default RS functionals. 
The computed vertical excitation energies from the ground state to the first excited state and are directly comparable to the optical gap observed experimentally from UV-vis absorption spectroscopy. The optical gaps of molecules computed using default RS functionals are plotted in Figure 6. Experimental values as well as the computed values of the optical gaps using B3LYP are also included in Figure 6 for comparison. As shown in Figure 6, the B3LYP results are very close to the experimental values with the MSE value of $\sim 0.02 \mathrm{eV}$. The small deviation of the optical gaps from the experimental values can be attributed to the lack of intermolecular interactions in the present computation that are present in the experimental measurements. All the RS functionals such as LC-BLYP, CAM-B3LYP and wB97XD with the default values of $w$ overestimate the optical gaps compared to the experimental values. However, the overestimation is not as severe as in the case of the HOMO-LUMO gaps shown in Figure 4. This is evident from MSE values of 0.47, 0.55 and $0.82 \mathrm{eV}$ for CAM-B3LYP, wB97XD and LC-BLYP, respectively. Nevertheless, the overall trend in the overestimation of optical gaps of molecules follows the trend of LC-BLYP > wB97XD > CAM-B3LYP, when default values of $w$ are adopted.

Table 3. Computed optical gap (eV) at LC-BLYP. Experimental values are from Refs. 1, 4, 34-39.

\begin{tabular}{cccccc}
\hline Molecule & Expt. & B3LYP & LC-BLYP (default) & LC-BLYP (tuned) & Optimal w \\
\hline M1 & 1.50 & 1.52 & 2.40 & 1.52 & 0.10 \\
M2 & 1.88 & 1.81 & 2.47 & 1.91 & 0.12 \\
M3 & 2.08 & 1.83 & 2.97 & 2.09 & 0.14 \\
M4 & 1.77 & 1.85 & 2.76 & 1.83 & 0.10 \\
M5 & 2.14 & 1.86 & 3.03 & 2.19 & 0.15 \\
M6 & 1.74 & 1.93 & 2.86 & 1.79 & 0.10 \\
M7 & 1.86 & 1.94 & 2.56 & 1.87 & 0.10 \\
M8 & 1.84 & 1.94 & 2.82 & 1.89 & 0.10 \\
M9 & 1.91 & 1.97 & 2.61 & 1.91 & 0.10 \\
M10 & 2.17 & 2.04 & 2.79 & 2.19 & 0.14 \\
M11 & 2.41 & 2.38 & 3.08 & 2.42 & 0.12 \\
M12 & 2.42 & 2.50 & 3.16 & 2.44 & 0.11 \\
\hline
\end{tabular}




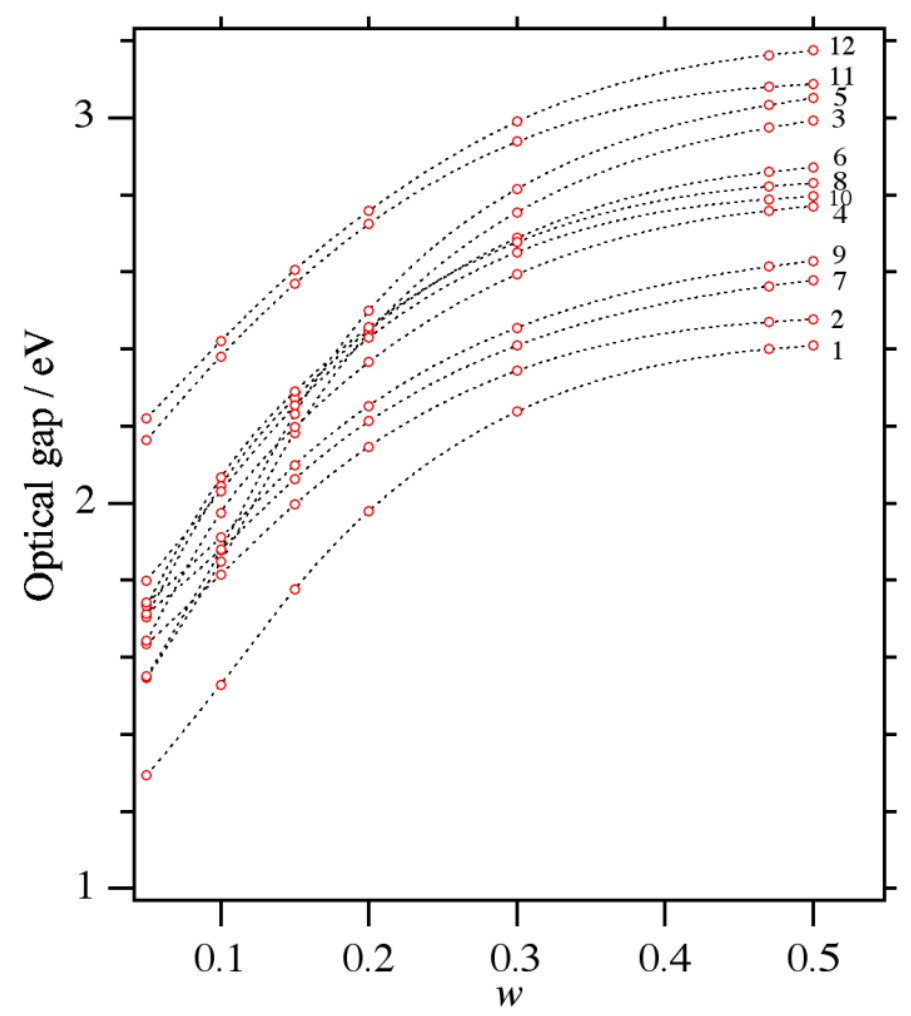

Figure 7. Variation of optical gaps of molecules as functions of $w$ for LC-BLYP.

The deviation of the computed optical gaps using default RS functionals from the experimental values can be eliminated by using the optimal values of $w$ for these functionals. The computed optical gaps for the twelve molecules using LC-BLYP with optimal values of $\mathrm{w}$ and the corresponding experimental data are presented in Table 3 . It is evident from Table 3 that the computed optical gaps using default and optimal values of $\mathrm{w}$ are significantly different from each other. Figure 7 shows the plot of the optical gaps of molecules as a function of $w$ for LC-BLYP. At the LC-BLYP level, the MSE value changes from $0.82 \mathrm{eV}$ to $-0.015 \mathrm{eV}$ when $w$ is tuned from $0.5 \mathrm{Bohr}^{-1}$ to $0.1 \mathrm{Bohr}^{-1}$. A further decrease in the value of $w$ to 0.05 gives the MSE value of $-0.24 \mathrm{eV}$. Both CAM-B3LYP and wB97XD RS functionals follow similar trends in which MSE values decrease to $0.02 \mathrm{eV}$ and $0.09 \mathrm{eV}$, respectively, as $w$ becomes equal to $0.05 \mathrm{Bohr}^{-1}$. This 
shows that the optimal values of $w$ to compute accurate optical gaps of these molecules fall between 0.1 and $0.15 \mathrm{Bohr}^{-1}$. Figure 8 shows the plot of the oscillator strengths of molecules as a function of $w$ for LC-BLYP. As found in the case of optical gap, the oscillator strengths decrease when $w$ is tuned from $0.5 \mathrm{Bohr}^{-1}$ to $0.05 \mathrm{Bohr}^{-1}$. CAMB3LYP and wB97XD RS functionals follow similar trends of oscillator strengths.

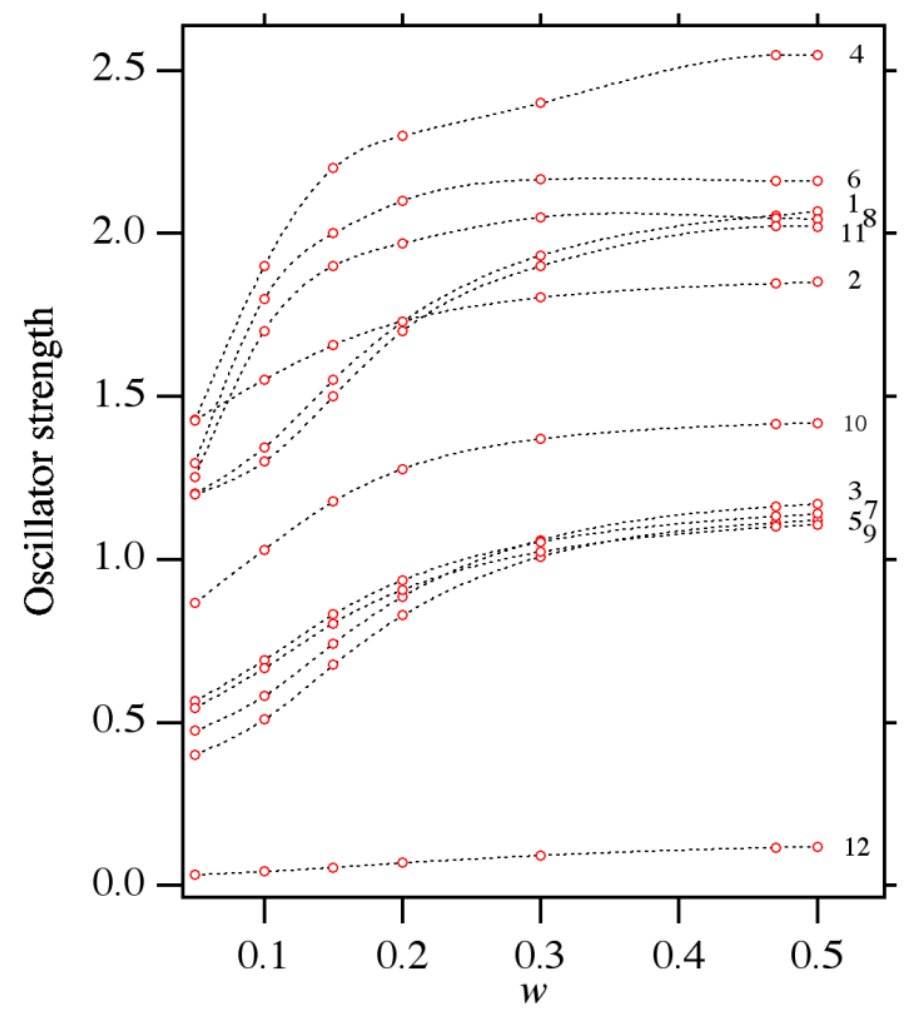

Figure 8. Variation of oscillator strengths of molecules as functions of $w$ for LCBLYP. Oscillator strengths of M4, M6 and M8 are multiplied by 0.5 to fit into the scale.

\section{Conclusions}

Orbital energy modeling of twelve representative conjugated molecules, including homo- as well as alternating donor-acceptor units was performed using DFT and TD- 
DFT methods. All of the ground state and the excited state DFT and TD-DFT calculations were carried out using B3LYP hybrid functionals as well as LC-BLYP, wB97XD and CAM-B3LYP RS functionals. Computations with the RS functionals were completed using both default and manually tuned range-separation parameters. For the range-separated functionals, the range-separated parameters were tuned in the range of $0.05 \mathrm{Bohr}^{-1}$ to $0.5 \mathrm{Bohr}^{-1}$.

We found that default RS functionals favors lower lying (more negative) HOMO and higher lying (less negative) LUMO energies compared to both B3LYP results and experimental values. Consequently, default RS functionals overestimate the HOMOLUMO as well as the vertical excitation energies. B3LYP is found to be more robust to predict accurate HOMO-LUMO and optical gaps than default RS functionals. LC-BLYP and $w B 97 X D$ functionals are found to be more sensitive to tuning the range-separation parameter as compared to CAM-B3LYP. Likewise, the average change in the HOMOLUMO gaps with the range-separation parameter is found to be greater than the optical gap. The present results show that accurate HOMO-LUMO and optical gaps of conjugated systems can be predicted using the RS functionals with range-separated parameter between 0.1 and $0.15 \mathrm{Bohr}^{-1}$. 


\section{Acknowledgements}

RSB and MT are grateful to the National Science Foundation (Grant No. DMR0847580) for financial support. This work used the extreme science and engineering discovery environment (XSEDE), which is supported by National Science Foundation Grant no. ACI-1053575. GP and MT acknowledge National Science Foundation of South Africa for financial Support (CPRR13090833946, Grant No. 92786). 


\section{References}

[1]. Y. Sun, G.C. Welch, W.L. Leong, C.J. Takacs, G.C. Bazan, A.J. Heeger, Solutionprocessed small-molecule solar cells with 6.7\% efficiency, Nat. Mater. 11 (2012) 4448.

[2]. L. Lin, Y. Chen, Z. Huang, H. Lin, S. Chou, F. Lin, C. Chen, Y. Liu, K. Wong, A low-energy-gap organic dye for high-performance small-molecule organic solar cells, J. Am. Chem. Soc. 133 (2011) 15822-15825.

[3]. J. Roncali, P. Leriche, P. Blanchard, Molecular materials for organic photovoltaics: small is beautiful, Adv. Mater. 26 (2014) 3821-3838.

[4]. Y. Liu, C. Chen, Z. Hong, J. Gao, Y. (Michael). Yang, H. Zhou, L. Dou, G. Li, Y. Yang, Solution-processed small-molecule solar cells: breaking the 10\% power conversion efficiency, Sci. Rep. 3 (2013) 3356.

[5]. J. You, L. Dou, K. Yoshimura, T. Kato, K. Ohya, T. Moriarty, K. Emery, C. Chen, J. Gao, G. Li, Y. Yang, A polymer tandem solar cell with $10.6 \%$ power conversion efficiency, Nat. Commun. 4 (2013) 1-10.

[6]. C. Liu, C. Yi, K. Wang, Y. Yang, R.S. Bhatta, M. Tsige, S. Xiao, X. Gong, Singlejunction polymer solar cells with over 10\% efficiency by a novel two-dimensional donor-acceptor conjugated copolymer, ACS Appl. Mater. Interfaces. 7 (2015) 49284935.

[7]. M.C. Scharber, D. Muhlbacher, M. Koppe, P. Denk, C. Waldauf, A.J. Heeger, C.J. Brabec, Design rules for donors in bulk-heterojunction solar cells-towards $10 \%$ energy-conversion efficiency, Adv. Mater. 18 (2006) 789-794. 
[8]. P.P. Khlyabich, B. Burkhart, B.C. Thompson, Compositional dependence of the open-circuit voltage in ternary blend bulk heterojunction solar cells based on two donor polymers, J. Am. Chem. Soc. 134 (2012) 9074-9077.

[9]. B. Burkhart, P.P. Khlyabich, B.C. Thompson, Influence of the Ethylhexyl Side-Chain Content on the Open-Circuit Voltage in rr-Poly(3-hexylthiophene-co-3-(2ethylhexyl)thiophene) Copolymers, Macromolecules, 45 (2012) 3740-3748.

[10]. P. Hohenberg, W. Kohn, Inhomogeneous electron gas, Phys, Rev. 136 (1964) B864-B871.

[11]. E. Runge, E.K.U. Gross, Density-functional theory for time-dependent systems, Phys. Rev. Lett. 52 (1984) 997-1000.

[12]. T.M. McCormick, C.R. Bridges, E.I. Carrera, P.M. DiCarmine, G.L. Gibson, J. Hollinger, L.M. Kozycz, D.S. Seferos, Conjugated polymers: evaluating DFT methods for more accurate orbital energy modeling, Macromolecules, 46 (2013) 3879-3886.

[13]. T. Korzdorfer, J. Bredas, Organic electronic materials: recent advances in the DFT description of the ground and excited states using tuned range-separated hybrid functionals, Acc. Chem. Res. 47 (2014) 3284-3291.

[14]. M.E. Foster, B.M. Wong, Nonempirically tuned range-separated DFT accurately predicts both fundamental and excitation gaps in DNA and RNA nucleobases, J. Chem. Theory Comput. 8 (2012) 2682-2687.

[15]. C. Zhang, J.S. Sears, B. Yang, S.G. Aziz, V. Coropceanu, J. Bredas, Theoretical study of the local and charge-transfer excitations in model complexes of 
pentacene- C60 using tuned range-separated hybrid functionals, J. Chem. Theory Comput. 10 (2014) 2379-2388.

[16]. M.J. Frisch, et al. Gaussian, Inc., Revision D.01, Wallingford, CT., USA, 2009.

[17]. T. Yanai, D. Tew, N. Handy, A new hybrid exchange-correlation functional using the coulomb-attenuating method (CAM-B3LYP), Chem. Phys. Lett. 393 (2004) 5157.

[18]. H. Iikura, T. Tsuneda, T. Yanai, K. Hirao, Long-range correction scheme for generalized-gradient-approximation exchange functionals, J. Chem. Phys. 115 (2001) 3540-3544.

[19]. J. Chai, M. Head-Gordon, Long-range corrected hybrid density functionals with damped atom-atom dispersion corrections, Phys. Chem. Chem. Phys. 10 (2008) 66156620.

[20]. R.S. Bhatta, M. Tsige, Understanding the effect of heteroatoms on structural and electronic properties of conjugated polymers, Polymer, 56 (2015) 293-299.

[21]. T. Stein, H. Eisenberg, L. Kronik, R. Baer, Fundamental gaps in finite systems from eigenvalues of a generalized Kohn-Sham method, Phys. Rev. Lett. 105 (2010) 266802-4.

[22]. S. Refaely-Abramson, R. Baer, L. Kronik, Fundamental and excitation gaps in molecules of relevance for organic photovoltaics from an optimally tuned rangeseparated hybrid functional, Phys. Rev. B 84 (2011) 075144-8.

[23]. L. Kronik, T. Stein, S. Refaely-Abramson, R. Baer, Excitation gaps of finite-sized systems from optimally tuned range-separated hybrid functionals, J. Chem. Theory Comput. 8 (2012) 1515-1531. 
[24]. B. M. Wong, J. G. Cordaro, Coumarin dyes for dye-sensitized solar cells: A longrange-corrected density functional study, J. Chem. Phys. 129 (2008) 214703-8.

[25]. B. M. Wong, T. H. Hsieh, Optoelectronic and excitonic properties of oligoacenes: substantial improvements from range-separated time-dependent density functional theory, J. Chem. Theory Comput. 6 (2010) 3704-3712.

[26]. S. Bonness, H. Fukui, K. Yoneda, R. Kishi, B. Champagne, E. Botek, M. Nakano, Theoretical investigation on the second hyperpolarizabilities of open-shell singlet systems by spin-unrestricted density functional theory with long-range correction: Range separating parameter dependence, Chem. Phys. Lett. 493 (2010) 195-199.

[27]. M. Srebro and J. Autschbach, Tuned range-separated time-dependent density functional theory applied to optical rotation, J. Chem. Theory Comput. 8 (2012) $245-$ 256.

[28]. S. Nenon, B. Champagne, M. I. Spassova, Assessing long-range corrected functionals with physically-adjusted range-separated parameters for calculating the polarizability and the second hyperpolarizability of polydiacetylene and polybutatriene chains, Phys. Chem. Chem. Phys., 16 (2014) 7083-7088.

[29]. S. Grimme, Semiempirical GGA-type density functional constructed with a longrange dispersion correction, J. Comput. Chem. 27 (2006) 1787-1799.

[30]. A.D. Becke, Density-functional thermochemistry. III. The role of exact exchange, J. Chem. Phys. 98 (1993) 5648-5652.

[31]. R.S. Bhatta, Y. Yimer, M. Tsige, D.S. Perry, Conformations and torsional potentials of poly(3-hexylthiophene) oligomers: Density functional calculations up to the dodecamer, Comput. Theor. Chem. 995 (2012) 36-42. 
[32]. R.S. Bhatta, D.S. Perry, Correlated backbone torsional potentials in poly(3methylthiophene), Comput. Theor. Chem. 1008 (2013) 90-95.

[33]. R.S. Bhatta, M. Tsige, Effect of fluorination on electronic properties of polythienothiophene-co-benzodithiophenes and their fullerene complexes, ACS Appl. Mater. Interfaces. 6 (2014) 15889-15896.

[34]. L. Lin, C. Lu, W. Huang, Y. Chen, H. Lin, K. Wong, New A-A-D-A-A-type electron donors for small molecule organic solar cells, Org. Lett. 13 (2011) 49624965.

[35]. Y. Chen, L. Lin, C. Lu, F. Lin, Z. Huang, H. Lin, P. Wang, Y. Liu, K. Wong, J. Wen, D.J. Miller, S.B. Darling, Vacuum-deposited small-molecule organic solar cells with high power conversion efficiencies by judicious molecular design and device optimization, J. Am. Chem. Soc. 134 (2012) 13616-13623.

[36]. J. Zhou, X. Wan, Y. Liu, Y. Zuo, Z. Li, G. He, G. Long, W. Ni, C. Li, X. Su, Y. Chen, Small molecules based on benzo[1,2-b:4,5-b']dithiophene unit for highperformance solution-processed organic solar cells, J. Am. Chem. Soc. 134 (2012) $16345-16351$.

[37]. J. Sakai, T. Taima, K. Saito, Efficient oligothiophene:fullerene bulk heterojunction organic photovoltaic cells, Org. Electron. 9 (2008) 582-590.

[38]. C. Chu, Y. Shao, V. Shrotriya, Y. Yang, Efficient photovoltaic energy conversion in tetracene-C60 based heterojunctions, Appl. Phys. Lett. 86 (2005) 243506-3.

[39]. N. Geacintov, M. Pope, F. Vogel, Effect of magnetic field on the fluorescence of tetracene crystals: exciton fission, Phys. Rev. Lett. 22 (1969) 593-596. 


\section{TOC:}
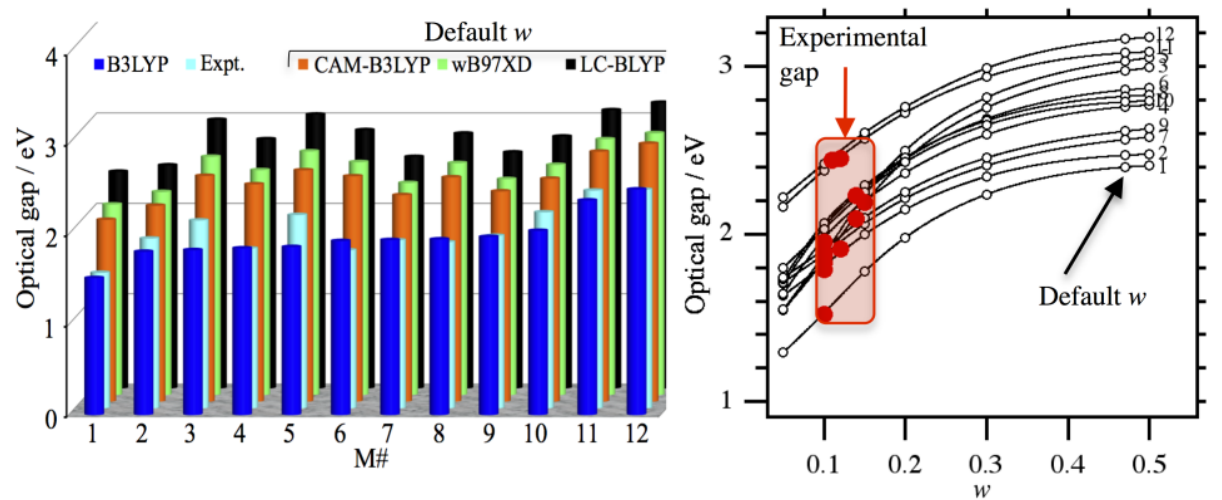